\title{
Predominance of Bacterial Flora Causing Lower Respiratory Tract Infections Following Tracheostomy in Patients of Chettinad Hospital \& Research Institute, Chennai, India
}

\author{
Swetha Thirumurthi ${ }^{1}$, Priya Kanagamuthu², Rajasekaran Srinivasan ${ }^{3}$, Bhalaji Dhanasekaran ${ }^{4}$ \\ 1, 2,3,4 Department of ENT, Chettinad Hospital and Research Institute, Kelambakkam, Tamil Nadu, India.
}

\section{ABSTRACT}

\section{BACKGROUND}

The term tracheostomy refers to forming an opening in the trachea.1,2 Its advantages include easy and direct access to lower respiratory tract, reduced risk of aspiration, faster weaning from ventilation support and improved physical and psychological comfort. But a common problem in tracheostomised patients is increased risk of colonisation of lower respiratory tract by exogenous bacteria because of direct exposure.1,3 This study was done to recognise pathogens in tracheal secretions collected from tracheostomised patients and their antibiotic sensitivity to treat them with appropriate antibiotics.

\section{METHODS}

This prospective study was done in 138 tracheostomised patients from October 2020 to March 2021 in intensive care unit (ICU) of Chettinad Hospital and Research Institute. Under sterile aseptic precautions, Day 0 and Day 7 cultures posttracheostomy was obtained and their antibiotic sensitivity was studied. Data was analysed using Statistical Package for Social Sciences (SPSS version 19) and presented in proportion, mean and standard deviation (Descriptive statistics).

\section{RESULTS}

In this study, of the 56 cases who had growth in their culture and sensitivity reports on day 0 , the most common organism was Pseudomonas aeruginosa (33.9\%) sensitive to imipenem ( $94.7 \%$ ) followed by klebsiella (25\%) sensitive to teicoplanin, vancomycin, amikacin, cefoperazone/tazobactam, linezolid and piperacillin/tazobactam. On day 7 , the growth of organisms isolated in tracheal culture got reduced from 56 cases to 16 cases. The prevalence of Pseudomonas reduced to $18.8 \%$ in day 7 whereas Klebsiella pneumonia and Acinetobacter remained almost same from day 0 to day 7 .

\section{CONCLUSIONS}

This study concludes the predominant pathogen as Pseudomonas aeruginosa with sensitivity to imipenem followed by Klebsiella with sensitivity to teicoplanin, vancomycin, amikacin, cefoperazone/tazobactam, linezolid and piperacillin/tazobactam on day 0 with reduction in the number of organisms on day 7 due to the fact that all our patients were admitted in ICU several days prior to tracheostomy and were started on antibiotics soon after admission as per choice of the treating physician. Hence, a clear understanding of bacterial colonisation post tracheostomy and its change in course is essential for timely intervention with empirical antibiotics for reducing the incidence of lower respiratory tract infections after tracheostomy in future.

\section{KEY WORDS}

Tracheostomy, Lower Respiratory Tract Infections, Pseudomonas Aeruginosa, Empirical Antibiotics.
Corresponding Author: Dr. Priya Kanagamuthu, Department of ENT, Chettinad Hospital and Research Institute, Kelambakkam, Tamil Nadu, India.

E-mail: catchpiya.29@gmail.com

DOI: $10.14260 /$ jemds/2021/606

How to Cite This Article:

Thirumurthi S, Kanagamuthu P, Srinivasan $R$, et al. Predominance of bacterial flora causing lower respiratory tract infections following tracheostomy in patients of Chettinad hospital \& research institute, Chennai, India. J Evolution Med Dent Sci 2021;10(35):2964-2968, 10.14260/jemds/2021/606

Submission 03-06-2021,

Peer Review 24-07-2021,

Acceptance 30-07-2021,

Published 30-08-2021.

Copyright (C) 2021 Swetha Thirumurthi et al. This is an open access article distributed under Creative Commons Attribution License [Attribution 4.0 International (CC $B Y 4.0)]$ 


\section{BACKGROUND}

Tracheostomy refers to creating a hole (Stoma) in the skin leading to the trachea in view of securing the airway. ${ }^{1,2}$ It's advantages include improved physical and psychological comfort to the patient, reduced risk of tube obstruction, improved clearance of tracheal secretions by suctioning, faster weaning from mechanical ventilation resulting in decreased ICU stay.4,5 Tracheostomy also reduces risk of developing ventilator associated pneumonia. ${ }^{6}$ Even though the benefits of tracheostomy outweigh its drawbacks, tracheostomy has its own risks and complications as well. Common problem that is being encountered in tracheostomised patients is increased risk of colonisation of lower respiratory tract by pathogens. 7,8

The normal trachea is protected from colonisation by bacteria, hence the trachea of healthy individuals have no bacteria. ${ }^{1}$ But following a tracheostomy, this protective role is partially bypassed and the lower respiratory tract is directly exposed to exogenous bacteria.1,9 However, due to inappropriate and inadequate use of antibiotics, bacterial resistance has become very common. ${ }^{10,6,11}$

\section{Aim}

This study aims to identify the nature of pathogen present in tracheal secretions in tracheostomised patients and analyse their antibiotic sensitivity in view of treating the lower respiratory tract infections following tracheostomy by timely intervention and treatment with empirical antibiotic therapy.

\section{Objectives}

Objectives of this study are as follows -

1. To obtain cultures from tracheal secretions of tracheostomised patients in day 0 and day 7 after tracheostomy.

2. To find the predominant bacteria in tracheal cultures and to study the change in their pattern over course of time.

3. To determine the antibiotic sensitivity for timely intervention with antibiotics to reduce the incidence of lower respiratory tract infections.

\section{METHODS}

After obtaining approval from ethical committee, this prospective cohort study was done in 138 tracheostomised patients in intensive care unit of Chettinad Hospital and Research Institute from October 2020 to March 2021(6 months). Using aseptic precautions, cultures were obtained from tracheal secretions using a sterile suction catheter, the tip of the suction catheter was cut and placed in a sterile container. The container was sealed and transported to the microbiology lab for bacteriological analysis. This was labelled as day 0 culture.

Standard post tracheostomy care including regular tracheobronchial suctioning using separate sterile suction catheters, 2 hourly deflation of the cuff for 10 min to reduce the risk of tracheal necrosis and granuloma, stomal dressing, provision of humidified air were done for all patients posttracheostomy.
On the seventh post-operative day, tracheal suctioning was done with a sterile suction catheter, its tip cut, put in a sterile container and sent for bacteriological analysis. This was day 7 culture. Bacterial cultures were done on MacConkey's agar/Chocolate agar/Sheep blood agar and the isolates were identified using grams staining and standard biochemical reactions.

\section{Inclusion Criteria}

All tracheostomised patients (elective/emergency) due to any cause.

\section{Exclusion Criteria}

- Patients already having an episode of lower respiratory tract infections (LRTI).

- Patients not willing for study

- Patients with comorbidities like diabetes, hypertension or any chronic disease.

The study was proceeded WITH after obtaining informed consent from all the patient's attenders.

\section{Statistical Analysis}

Data was analysed using Statistical Package for Social Sciences version 19. Data was presented in proportion, mean and standard deviation (Descriptive statistics).

\section{RESULTS}

In this study, of the 138 participants, majority of the study participants $(24.6 \%)$ belonged to age group between $41-50$ years followed by 51 - 60 years $(20.3 \%), 31$ - 40 years (18.8 \%), 61 - 70 years (14.5\%), more than 70 years (10.9\%), 21 30 years $(8.7 \%)$ and $\leq 20$ years $(2.2 \%)$. Mean age of the study participants was found to be $46.6 \pm 21.6$ years, in this study. Also, in this study, $57.2 \%$ and $42.8 \%$ of the study participants were males and females, respectively. On assessing the indications for tracheostomy, there were $5.8 \%, 22.5 \%$ and $12.3 \%$ of cases who underwent tracheostomy due to carcinoma of head and neck region, polytrauma and respiratory failure, respectively. Rest of the cases underwent tracheostomy following major surgeries. In this study, $25.4 \%$, $15.9 \%$ and $18.1 \%$ of cases underwent tracheostomy following neurosurgical procedures, cardiothoracic procedures and abdominal surgeries, respectively. In the present study, 40.6\% of cases showed growth of micro-organisms and $59.4 \%$ of cases showed no growth of micro-organisms in their culture and sensitivity reports.

Among the 56 cases those who had growth in their culture and sensitivity reports, $33.9 \%, 25 \%, 17.9 \%, 7.1 \%, 5.4 \%, 3.6$ $\%, 5.4 \%$ and $1.8 \%$ of the cases had Pseudomonas aeruginosa, Klebsiella pneumonia, Staphylococcus aureus, Methicillin resistant Staphylococcus aureus, Streptococcus pneumonia, Proteus mirabilis, Escherichia coli and Acinetobacter, respectively (Table 1 ). 


\begin{tabular}{|ccc|}
\hline Organisms & $\begin{array}{c}\text { Frequency } \\
\text { N = 56 }\end{array}$ & Percentage \\
\hline Pseudomonas aeruginosa & 19 & 33.9 \\
Klebsiella pneumonia & 14 & 25 \\
Staphylococcus aureus & 10 & 17.9 \\
Methicillin resistant staphylococcus aureus & 04 & 7.1 \\
Streptococcus pneumonia & 03 & 5.4 \\
Proteus mirabilis & 02 & 3.6 \\
Escherichia coli & 03 & 5.4 \\
Acinetobacter & 01 & 1.8 \\
\hline Table 1. Proportion of Cases with Growth in Culture on Day 0 \\
\hline
\end{tabular}

\begin{tabular}{|cccc|}
\hline Antibiotics & \multicolumn{3}{|c|}{ Pseudomonas sp. (N = 19) } \\
& $\begin{array}{c}\text { No. of Sensitive } \\
\text { Cases (\%) }\end{array}$ & $\begin{array}{c}\text { No. of Resistant } \\
\text { Cases (\%) }\end{array}$ & $\begin{array}{c}\text { No. of } \\
\text { Intermittent } \\
\text { Cases (\%) }\end{array}$ \\
GEN & $13(68.4)$ & $6(31.6)$ & $0(0)$ \\
TOB & $12(63.2)$ & $6(31.6)$ & $1(5.3)$ \\
PIT & $14(73.7)$ & $4(21.1)$ & $1(5.3)$ \\
AK & $10(52.6)$ & $8(42.1)$ & $1(5.3)$ \\
LOM & $6(31.6)$ & $11(57.9)$ & $2(10.5)$ \\
AS & $9(47.4)$ & $7(36.8)$ & $3(15.8)$ \\
LE & $9(47.4)$ & $9(47.4)$ & $1(5.3)$ \\
IPM & $18(94.7)$ & $1(5.3)$ & $0(0)$ \\
MRP & $17(89.5)$ & $2(10.5)$ & $0(0)$ \\
CPT & $13(68.4)$ & $5(26.3)$ & $2(10.5)$ \\
CPM & $14(73.7)$ & $4(21.1)$ & $1(5.3)$ \\
\hline CFS & $11(57.9)$ & $8(42.1)$ & $0(0)$ \\
TCC & $5(26.3)$ & $13(68.4)$ & $1(5.3)$ \\
\hline Table 2. Sensitivity Pattern of Pseudomonas Species on Day 0 \\
\hline \multicolumn{4}{r}{}
\end{tabular}

\begin{tabular}{|c|c|c|c|}
\hline \multirow[b]{2}{*}{ Antibiotics } & \multicolumn{3}{|c|}{ Klebsiella sp. $(\mathrm{N}=14)$} \\
\hline & $\begin{array}{l}\text { No. of Sensitive } \\
\text { Cases (\%) }\end{array}$ & $\begin{array}{l}\text { No. of Resistant } \\
\text { Cases (\%) }\end{array}$ & $\begin{array}{c}\text { No. of } \\
\text { Intermittent } \\
\text { Cases (\%) }\end{array}$ \\
\hline LE & $9(64.3)$ & $4(28.6)$ & $1(7.1)$ \\
\hline MO & 8 (57.1) & $6(42.9)$ & $0(0)$ \\
\hline LOM & $6(42.9)$ & 8 (57.1) & $0(0)$ \\
\hline TOB & $12(85.7)$ & $2(14.3)$ & $0(0)$ \\
\hline TEI & $13(92.9)$ & $0(0)$ & $1(7.1)$ \\
\hline VA & $13(92.9)$ & $1(7.1)$ & $0(0)$ \\
\hline DO & $14(100)$ & $0(0)$ & $0(0)$ \\
\hline G & $12(85.7)$ & $2(14.3)$ & $0(0)$ \\
\hline $\mathrm{AK}$ & $13(92.9)$ & $0(0)$ & $1(7.1)$ \\
\hline $\mathrm{E}$ & $11(78.6)$ & $2(14.3)$ & $1(7.1)$ \\
\hline A & $8(57.1)$ & $4(28.6)$ & $2(14.3)$ \\
\hline CPT & $11(78.6)$ & $2(14.3)$ & $1(7.1)$ \\
\hline CFS & $13(92.9)$ & $1(7.1)$ & $0(0)$ \\
\hline CXM & $10(71.4)$ & $3(21.4)$ & $1(7.1)$ \\
\hline CPM & $9(64.3)$ & $5(35.7)$ & $0(0)$ \\
\hline LZ & $13(92.9)$ & $1(7.1)$ & $0(0)$ \\
\hline PIT & $13(92.9)$ & $0(0)$ & $1(7.1)$ \\
\hline
\end{tabular}

\begin{tabular}{|cccc|}
\hline Antibiotics & \multicolumn{3}{|c|}{ Staphylococcus sp. (N = 14) } \\
& $\begin{array}{c}\text { No. of Sensitive } \\
\text { Cases (\%) }\end{array}$ & $\begin{array}{c}\text { No. of Resistant } \\
\text { Cases (\%) }\end{array}$ & $\begin{array}{c}\text { No. of } \\
\text { Intermittent } \\
\text { Cases (\%) }\end{array}$ \\
LE & $8(57.1)$ & $4(28.6)$ & $2(14.3)$ \\
MO & $8(57.1)$ & $6(42.9)$ & $0(0)$ \\
LOM & $6(42.9)$ & $8(57.1)$ & $0(0)$ \\
TOB & $12(85.7)$ & $2(14.3)$ & $0(0)$ \\
TEI & $13(92.9)$ & $1(7.1)$ & $0(0)$ \\
VA & $14(100)$ & $0(0)$ & $0(0)$ \\
DO & $14(100)$ & $0(0)$ & $0(0)$ \\
G & $12(85.7)$ & $2(14.3)$ & $0(0)$ \\
AK & $13(92.9)$ & $1(7.1)$ & $0(0)$ \\
E & $10(71.4)$ & $2(14.3)$ & $2(14.3)$ \\
CPT & $1178.6)$ & $1(7.1)$ & $2(14.3)$ \\
CFS & $13(92.9)$ & $1(7.1)$ & $0(0)$ \\
CXM & $10(71.4)$ & $3(21.4)$ & $1(7.1)$ \\
\hline CPM & $11(78.6)$ & $3(21.4)$ & $0(0)$ \\
LZ & $13(92.9)$ & $1(7.1)$ & $0(0)$ \\
PIT & $13(92.9)$ & $0(0)$ & $1(7.1)$ \\
\hline Table 4. Sensitivity Pattern of Staphylococcus Species on Day 0 \\
\hline \multicolumn{4}{|c}{}
\end{tabular}

Sensitivity pattern of Pseudomonas species, Klebsiella species and Staphylococcus species is shown in the Table 2, Table 3 and Table 4, respectively.

PIT: Piperacillin - Tazobactam; CPM: Cefepime; CPT: Cefepime / Tazobactam;

CXM: Cefuroxime; CFS: Cefoperazone/Tazobactam; G: Gentamycin; CX: Cefoxitin;
AK: Amikacin; TOB: Tobramycin; AS: Ampicillin/Sulbactam; LZ: Linezolid;

LE: Levofloxacin; A: Ampicillin; DO: Doxycycline; MO: Moxifloxacin, E: Erythromycin;

VA: Vancomycin; TEI: Teicoplanin; LOM: Lomefloxacin, IP: Imipenem,

MRP: Meropenem, CFS: Cefoperazone/Tazobactam

On day 7 of culture and sensitivity, $16(11.5 \%)$ cases showed growth of micro-organisms and 122 (88.4\%) cases showed no growth of micro-organisms in their culture and sensitivity reports. Among the 16 cases with growth of culture and sensitivity of day 7, there were $18.8 \%, 25 \%, 18.8 \%, 18.8$ $\%, 6.3 \%$ and $12.5 \%$ of the cases had Pseudomonas aeruginosa Klebsiella pneumonia, Staphylococcus aureus, Methicillin resistant Staphylococcus aureus, Proteus mirabilis and Acinetobacter respectively (Table 5).

\begin{tabular}{|cc|c|}
\hline Organisms & $\begin{array}{c}\text { Frequency } \\
\text { (N = 16) }\end{array}$ & Percentage \\
\hline Pseudomonas aeruginosa & 03 & 18.8 \\
Klebsiella pneumonia & 04 & 25 \\
Staphylococcus aureus & 03 & 18.8 \\
Methicillin resistant Staphylococcus aureus & 03 & 18.8 \\
Proteus mirabilis & 01 & 6.3 \\
Acinetobacter & 02 & 12.5 \\
\hline Table 5. Proportion of Cases with Growth in Culture on Day 7 \\
\hline
\end{tabular}

\section{DISCUSSION}

The trachea of healthy individuals is sterile and protected from bacterial colonisation. After tracheostomy, the protective mechanism is partially bypassed causing direct exposure of lower respiratory tract to exogenous bacteria. ${ }^{1}$ This happens mainly because the filtering mechanism of upper airways is lost after a tracheostomy, reduced effectiveness of cough reflex and ineffective closure of glottis paving way to aspiration. These are the factors which lead to bacterial colonisation and lower respiratory tract infections in tracheostomised patients. This study helps to identify bacterial flora in lower respiratory tract during tracheostomy and the change in the flora over time. This knowledge will help the treating physician in choosing empirical antibiotics in view of preventing and treating lower respiratory tract infections post tracheostomy. $15,13,8,16$

In our study, majority of the study participants belonged to age group 41 to 50 years with almost equal predisposition of males and females and with varied indications of tracheostomy. In our study, among the 56 cases who had growth in their culture and sensitivity reports as early as day 0 of culture, Pseudomonas aeruginosa was predominant (33.9 $\%$ ) with most sensitivity to imipenem. Second most common organism was Klebsiella pneumonia (25\%) with sensitivity to teicoplanin, vancomycin, cefoperazone/tazobactam, amikacin, piperacillin/tazobactam and linezolid followed by Staphylococcus aureus with sensitivity to vancomycin and doxycycline. This is due to the fact that many of our patients were hospitalised many days prior to tracheostomy resulting in nosocomial infections that might have led to the predominance of these pathogens as early as day 0 of culture and also due to the fact that antibiotics were not prescribed by the operating surgeon before or after tracheostomy. But 
antibiotics which were already prescribed by the treating physician were continued post tracheostomy.

In literature, the predominant organism isolated in tracheal aspirates is Pseudomonas aeruginosa similar to our study. ${ }^{7}$ In a study conducted by Aswin Mukundan ${ }^{1} \mathrm{M}$ Panduranga Kamath, ${ }^{4}$ Shrikala Baliga, ${ }^{7}$ Vijendra Shenoy S, ${ }^{10}$ Kiran M Bhojwani,12 Vishnu Prasad. ${ }^{5}$ there was almost negligible tracheal colonisation in day 0 culture yielding a negative growth in $94.6 \%$ of cases with very few samples showing growth of Acinetobacter (3.9\%) and Klebsiella species(1.5\%) respectively where as in our study many patients who underwent tracheostomy had pathogens isolated from tracheal cultures as early as day 0 of culture. This is because of the fact that most of our patients who underwent tracheostomy were critically ill and hospitalised many days prior to tracheostomy leading to the possibility of nosocomial infections. ${ }^{10}$

In literature, the predominant pathogens causing hospital acquired infections were found to be gram negative organisms predominantly Pseudomonas and Acinetobacter species similar to our study where colonisation by Pseudomonas was present as early as day 0 of culture (33.9\%) which reduced to $18.8 \%$ on day 7 , but colonisation by Acinetobacter species had increased from $1.8 \%$ on day 0 to $12.5 \%$ on day 7 proving Acinetobacter species to be hospital acquired and multidrugresistant as present in literature. ${ }^{10}$ In yet another study by Aliskan et al. higher risk of colonisation by potential multidrug-resistant organisms especially Pseudomonas aeruginosa and Acinetobacter species is being mentioned. ${ }^{14}$ In another recent study done by Cardenosa et al. most of the patients who were previously intubated and who were already on antibiotics showed heavy growth of Pseudomonas and Acinetobacter species. ${ }^{5}$

Recent studies have also implicated the role of bacterial biofilms on tracheostomy tubes which we have not studied here, which could be a major cause of persistent infection in this era of strict aseptic care and higher antibiotics. ${ }^{15,17,18,19}$

\section{CONCLUSIONS}

Colonisation of lower respiratory tract after tracheostomy is mainly by exogenous route. It is very essential to follow the patients by regular tracheal cultures. The main pathogens involved in tracheal colonisation in our study were Pseudomonas aeruginosa, Klebsiella pneumonia, Staphylococcus aureus and Acinetobacter species on day 0 with Acinetobacter species being predominant in previously intubated patients and patients on prolonged ICU stay proving that nosocomial infections are predominant in such patients. The number of organisms in tracheal culture reduced in day 7 due to the fact that all our patients were admitted in ICU several days prior to tracheostomy and were started on antibiotics soon after admission as per choice of the treating physician.

\section{Limitations of This Study}

Our study had its own limitations, but we were able to understand the change in the course of bacterial flora from day 0 post tracheostomy to day 7 . Predominant pathogen in day 0 culture was Pseudomonas which was sensitive to imipenem where as in day 7 culture the colonisation by Pseudomonas reduced. Even though the overall percentage of positive culture reports reduced in day 7 , the pathogens which were persistent were found to be Acinetobacter and Klebsiella species. Hence, a clear understanding of bacterial colonisation post tracheostomy and its change in course is essential to start on timely intervention with empirical antibiotics for reducing the incidence of lower respiratory tract infections after tracheostomy in future. $20,21,22,23$

\section{Recommendations}

Hence a clear understanding of bacterial colonisation post tracheostomy and its change in course is essential for timely intervention with empirical antibiotics for reducing the incidence of lower respiratory tract infections after tracheostomy in future.

Data sharing statement provided by the authors is available with the full text of this article at jemds.com.

Financial or other competing interests: None.

Disclosure forms provided by the authors are available with the full text of this article at jemds.com.

I would like to acknowledge all the members who guided and supported me to complete the study.

Ethical approval: This study was registered with Institutional Human Ethical Committee.

\section{REFERENCES}

[1] Laurenzi GA, Potter RT, Kass EH. Bacteriologic flora of the lower respiratory tract. New England Journal of Medicine 1961;265(26):1273-8.

[2] Morar P, Singh V, Makura Z, et al. Differing pathways of lower airway colonization and infection according to mode of ventilation (endotracheal vs. tracheotomy). Archives of Otolaryngology-Head \& Neck Surgery 2002;128(9):1061-6.

[3] Niederman MS, Ferranti RD, Zeigler A, et al. Respiratory infection complicating long-term tracheostomy: the implication of persistent gram-negative tracheobronchial colonization. Chest 1984;85(1):39-44.

[4] Ahmed QA, Niederman MS. Respiratory infection in the chronically critically ill patient: ventilator-associated pneumonia and tracheobronchitis. Clinics in Chest Medicine 2001;22(1):71-85.

[5] Cendrero JA, Solé-Violán J, Benitez AB, et al. Role of different routes of tracheal colonization in the development of pneumonia in patients receiving mechanical ventilation. Chest 1999;116(2):462-70.

[6] Brook I. Bacterial colonization, tracheobronchitis and pneumonia following tracheostomy and long-term intubation in pediatric patients. Chest 1979;76(4):420-4.

[7] Sakurai S, Ono T, Amanai T, et al. Detection of Pseudomonas aeruginosa following tracheostomy. Oral Therapeutics and Pharmacology 2005;24(1):7-12.

[8] Harlid R, Andersson G, Frostell CG, et al. Respiratory tract colonization and infection in patients with chronic tracheostomy. A one-year study in patients living at home. American Journal of Respiratory and Critical Care Medicine 1996;154(1):124-9. 
[9] Friedberg SA, Griffith TE, Hass GM. LXIV Histologic changes in the trachea following tracheostomy. Annals of Otology, Rhinology \& Laryngology 1965;74(3):785-98.

[10] Jung JY, Park MS, Kim SE, et al. Risk factors for multi-drug resistant Acinetobacter baumannii bacteremia in patients with colonization in the intensive care unit. BMC Infect Dis 2010;10:228.

[11] Bartlett JG, Faling LJ, Willey S. Quantitative tracheal bacteriologic and cytologic studies in patients with longterm tracheostomies. Chest 1978;74(6):635-9.

[12] Alişkan H, Colakoğlu S, Turunç T, et al. Four years of monitoring of antibiotic sensitivity rates of pseudomonas aeruginosa and Acinetobacter baumannii strains isolated from patients in intensive care unit and inpatient clinics. Mikrobiyoloji Bulteni 2008;42(2):321-9.

[13] Siddiqui ZR, Ahmed E, Niaz - Ud - Din, et al. Antimicrobial sensitivity of lower respiratory tract infections in tracheostomised severe head injury patients. Ann Pak Inst Med Sci 2011;7(2):52-6.

[14] Afolabi-Brown O, Marcus M, Speciale $P$, et al. Bronchoscopic and nonbronchoscopic methods of airway culturing in tracheostomized children. Respir Care 2014;59(4):582-7.

[15] Solomon DH, Wobb J, Buttaro BA, et al. Characterization of bacterial biofilms on tracheostomy tubes. The Laryngoscope 2009;119(8):1633-8.

[16] Pignatti P, Balestrino A, Herr C, et al. Tracheostomy and related host-patogen interaction are associated with airway inflammation as characterized by tracheal aspirate analysis. Respiratory Medicine 2009;103(2):201-8.
[17] Craven DE, Lei $Y$, Ruthazer $R$, et al. Incidence and outcomes of ventilator-associated tracheobronchitis and pneumonia. The American Journal of Medicine 2013;126(6):542-9.

[18] Morar P, Singh V, Makura Z, et al. Oropharyngeal carriage and lower airway colonisation/infection in 45 tracheotomised children. Thorax 2002;57(12):1015-20.

[19] Bhatta DR, Hamal D, Shrestha R, et al. Burden of multidrug resistant respiratory pathogens in intensive care units of tertiary care hospital. Asian Journal of Medical Sciences 2019;10(2):14-9.

[20] Koirala P, Bhatta RD, Ghimire P, et al. Bacteriological profile of tracheal aspirates of the patients attending a neuro-hospital of Nepal. Int J Life Sci 2010;4:60-5.

[21] Akingbade OA, Ogiogwa JI, Okerentugba PO, et al. Prevalence and antibiotic susceptibility pattern of bacterial agents involved in lower respiratory tract infections in Abeokuta, Ogun State, Nigeria. Report and Opinion 2012;4(5):25-30.

[22] Chawla K, Vishwanath S, Munim FC. Nonfermenting gramnegative bacilli other than pseudomonas aeruginosa and acinetobacter spp. causing respiratory tract infections in a tertiary care center. J Glob Infect Dis 2013;5(4):144-8.

[23] Regha IR, Sulekha B. Bacteriological profile and antibiotic susceptibility patterns of lower respiratory tract infections in a tertiary care hospital, Central Kerala. Int J Med Microbiol Tropical Dis 2018;4(4):186-90. 